\title{
HERBICIDES FOR GENTIANS
}

\author{
A. INGLE and G.K. BURGE \\ New Zealand Institute for Crop \& Food Research Ltd, Private Bag 4005, Levin
}

\begin{abstract}
Gentians are a small but expanding flower crop in New Zealand. Few herbicide recommendations are available for gentians. A range of pre-emergence herbicides were tested, with application in August when the buds on the crown were still dormant. Established crops appeared to tolerate herbicides better than newly planted crops. Herbicide mixtures with low rates of simazine or terbuthylazine combined with low rates of alachlor or diuron gave good weed control without damage to an established crop. Post-emergence application of haloxyfop at $0.25 \mathrm{~kg} / \mathrm{ha}$ gave good control of summer grasses and did not damage the crop.
\end{abstract}

Keywords: gentians, weed control, herbicide tolerance

\section{INTRODUCTION}

Gentians are an important flower crop in Japan, and over the last 15 years there has been increasing international interest in this crop. The main cultivars grown are interspecific hybrids between Gentiana triflora var. japonica, G. makinoi and G. scabra var. buergeri subvar. orientalis. This crop has been grown in New Zealand over the last decade, but stem quality and production problems have limited the expansion of the crop.

Gentians develop a crown with many buds which are dormant in winter. The crop seems well-suited to the use of pre-emergence herbicides in late winter. However, there are few publications about the use of herbicides on this crop. Batt (1982) indicated that simazine was safe on gentian crops and Barralis et al. (1978) found two amide herbicides (propachlor and diphenamid) to be safe on G. lutea. In a preliminary trial (Ingle unpublished) several herbicides (e.g. simazine, metolachlor) appeared to be safe on this crop. This paper outlines the results of three experiments evaluating residual herbicides, including two post-emergence grass herbicides, on gentians.

\section{METHODS}

The gentians (G. triflora var. japonica-like) used in all three trials were planted at the Levin Research Centre on 19 August 1993, on land previously cropped with squash. The soil type was Levin silt loam with a $\mathrm{pH}$ of 5.5. Two year old gentian crowns were planted with the buds just below the soil surface in $0.85 \mathrm{~m}$ wide raised beds with two rows of plants $0.4 \mathrm{~m}$ apart and plants spaced $0.4 \mathrm{~m}$ apart within the row. A $3 \mathrm{~m}$ bed length was used for each plot in all experiments, with a $1 \mathrm{~m}$ guard area between plots.

\section{Trial 1}

Eleven herbicide treatments were compared to an untreated control in a randomised block design with four replicates. Treatments were applied on 27 August 1993 using an Oxford Precision sprayer, spray volume of 350 litres/ha and pressure of $193 \mathrm{kPa}$. Weeds were identified and counted in three quadrats of $30 \times 30 \mathrm{~cm}$ per plot on 21 September, and on 20 October plots were scored for weed density using a 1-10 scale (Table 1). All plots were hand-weeded on 26 October; the grasses were not removed from one of the treatments (simazine followed by fluazifop-butyl), as the latter is a post-emergence grass herbicide. The fluazifop-butyl was applied on 9 November with the same volume of water and pressure as used with the earlier herbicide treatments. All plots were hand-weeded again on 20 December 1993. The number and length of gentian stems were recorded on 12-13 January 1994, and all plants were scored for leaf necrosis and leaf purpling. 


\section{Trial 2}

Some of the more promising herbicides from Trial 1 and other combinations were assessed in the subsequent season. This trial area was treated with simazine $(1.5 \mathrm{~kg} /$ ha) in the previous spring and was hand-weeded during the summer and winter. Treatments were replicated four times in a randomised block design. The treatments were applied on 3 August 1994 using a Mesto compressed air sprayer fitted with a Hardi boom with four jets, at a pressure of $150 \mathrm{kPa}$ and volume of 355 litres/ha. Weeds were identified and counted in four quadrats per plot on 6 September and plots were scored for weed density on 11 October. The control plots were hand-weeded on 20 October and on 15 December all plots were hand-weeded but the grasses were left intact in the simazine followed by haloxyfop treatment. The haloxyfop was applied on 23 December and the control of grasses was assessed on 12 January. Plants were scored for leaf symptoms (chlorosis, necrosis, purpling) on 21 October and for leaf purpling on 31 January when the stems were flowering. Stem number and length were measured in early February.

Trial 3

More persistent herbicides were assessed in this trial. The gentian crowns were covered with $6 \mathrm{~cm}$ of soil so that the herbicides would not contact the buds. Treatments were replicated twice in a randomised block design. The treatments were applied on 19 August 1994 in the same way as in Trial 2 and the granular herbicides were applied with a pepper pot sprinkler. The trial was hand-weeded and assessed as in Trial 2.

\section{TABLE 1: Trial 1. Weed numbers on 21 September, weed score on 20 October and the main weed species not controlled by the herbicide treatments on a recently planted gentian crop.}

\begin{tabular}{|c|c|c|c|c|c|}
\hline $\begin{array}{l}\text { Herbicide treatment }{ }^{1} \\
\text { and rate }(\mathrm{kg} \text { ai } / \mathrm{ha})\end{array}$ & $\begin{array}{c}\text { Weed } \\
\text { count } \\
\left(\text { No. } / \mathrm{m}^{2}\right)\end{array}$ & $\begin{array}{l}\text { Weed } \\
\text { score }^{3}\end{array}$ & Main weed species & $\begin{array}{c}\text { Mean } \\
\text { stem } \\
\text { length } \\
(\mathrm{cm})\end{array}$ & $\begin{array}{c}\text { Leaf } \\
\text { necrosis } \\
\text { score }^{6}\end{array}$ \\
\hline control & 285 & 8.75 & twin cress ${ }^{4}$, fumitory & 48.3 & 1.5 \\
\hline terbuthylazine 0.67 & 30 & 2.5 & twin cress, nightshade & 45.8 & 2.0 \\
\hline terbuthylazine 1.0 & 24 & 2.0 & twin cress, nightshade & 43.7 & 2.5 \\
\hline simazine 1.0 & 27 & 3.0 & twin cress, fumitory & 45.4 & 2.1 \\
\hline simazine 1.5 & 38 & 2.75 & twin cress, fumitory & 40.4 & 2.1 \\
\hline simazine 2.25 & 13 & 2.25 & twin cress, fumitory & 38.9 & 3.3 \\
\hline alachlor 2.0 & 45 & 5.0 & twin cress, fumitory & 44.4 & 1.8 \\
\hline $\begin{array}{l}\text { metolachlor } 2.0 \\
\text { simazine } 1.0\end{array}$ & 84 & 5.25 & twin cress, fumitory & 43.9 & 2.0 \\
\hline $\begin{array}{l}+ \text { alachlor } 1.0 \\
\text { simazine } 1.0\end{array}$ & 19 & 1.75 & -5 & 40.0 & 2.5 \\
\hline $\begin{array}{l}+ \text { metolachlor } 1.0 \\
\text { simazine } 1.0\end{array}$ & 21 & 2.0 & - & 42.8 & 1.7 \\
\hline $\begin{array}{l}+ \text { diuron } 0.4 \\
\text { simazine } 1.0 /\end{array}$ & 20 & 2.25 & fumitory & 44.0 & 2.0 \\
\hline fluazifop-butyl 0.4 & -2 & -2 & twin cress, fumitory & 41.3 & 2.0 \\
\hline LSD $5 \%$ & 118 & 1.0 & 4.0 & 0.8 & \\
\hline
\end{tabular}

${ }^{1}$ terbuthylazine (Gardoprim), simazine (Gesatop), alachlor (Shell alachlor), metolachlor (Dual), diuron (Karmex), fluazifop-butyl (Fusilade). ${ }^{2}$ Not recorded as fluazifopbutyl, applied after the time of this weed count and weed score. ${ }^{3}$ Plots were scored on a 0 -10 scale: $0=$ no weeds, $3=$ several weeds per plot, $5=10 \%$ weed cover, $7=20 \%$ weed cover, $10=70 \%$ weed cover (weediest plot). ${ }^{4}$ twin cress (Coronopus didymus), fumitory (Fumaria muralis), nightshade (Solanum nigrum). ${ }^{5}$ No species dominant. ${ }^{6}$ Plants were scored on a $0-10$ scale: $0=$ no symptoms, $10=$ plants with $50 \%$ necrotic leaves. 


\section{Trial 1}

\section{RESULTS}

All herbicide treatments significantly reduced weed density (Table 1). Simazine or terbuthylazine at the higher rates, or simazine combined with alachlor, metolachlor or diuron gave the best weed control. Fluazifop-butyl gave very good control of summer grass and smooth summer grass (Digitaria sanguinalis and D. ischaemum) but failed to control annual poa (Pоа апnиа).

There were no significant differences between treatments in the number of stems per plant (data not shown). However, stem length was significantly reduced by some of the herbicide treatments. The greatest reduction in stem length was with the higher rates of simazine, simazine + fluazifop-butyl and simazine + alachlor treatments (Table 1).

There was no significant difference in leaf purpling between the treatments (data not shown). Leaf necrosis was significantly increased by the higher rate of terbuthylazine and simazine, and simazine + alachlor.

\section{Trial 2}

All herbicide treatments gave almost complete weed control in September and October (Table 2). Haloxyfop controlled both species of summer grass and did not result in any symptoms of leaf disorders. There were no significant differences between the treatments either for leaf symptoms or for the plant measurements.

TABLE 2: Trial 2. Weed count on 6 September, weed score on 11 October, leaf symptoms score on 21 October, leaf purpling score on 31 January and mean stem number and length in early February, on an established gentian block.

\begin{tabular}{|c|c|c|c|c|c|c|}
\hline $\begin{array}{l}\text { Herbicide treatment } \\
\text { and rate }(\mathrm{kg} \text { ai } / \mathrm{ha})\end{array}$ & $\begin{array}{c}\text { Weed } \\
\text { count } \\
\left(\mathrm{No} . / \mathrm{m}^{2}\right)\end{array}$ & $\begin{array}{l}\text { Weed } \\
\text { score }^{2}\end{array}$ & $\begin{array}{c}\text { Score } \\
\text { for } \\
\text { leaf } \\
\text { symptoms }\end{array}$ & $\begin{array}{c}\text { Leaf } \\
\text { purpling } \\
\text { score }^{6}\end{array}$ & $\begin{array}{c}\text { Mean } \\
\text { stem } \\
\text { number } \\
\text { per plant }\end{array}$ & $\begin{array}{l}\text { Mean } \\
\text { stem } \\
\text { length } \\
(\mathrm{cm})\end{array}$ \\
\hline control & 82 & 6.75 & 2.7 & 3.9 & 10.1 & 39.1 \\
\hline simazine $1.0+$ alachlor 1.0 & $0 \quad 8$ & $0.25^{3}$ & 2.5 & 3.8 & 11.2 & 40.5 \\
\hline simazine $1.5+$ alachlor 0.5 & 5 & 0 & 2.7 & 3.7 & 10.3 & 43.3 \\
\hline simazine $1.5+$ alachlor 1.0 & 3 & 0 & 3.0 & 3.7 & 8.8 & 38.2 \\
\hline simazine 2.5 & 4 & $0.25^{3}$ & 3.0 & 3.8 & 8.8 & 41.9 \\
\hline $\begin{array}{l}\text { terbuthylazine } 0.6 \\
\quad+\text { alachlor } 1.0 \\
\text { terbuthylazine } 0.9\end{array}$ & 5 & $0.25^{4}$ & 2.9 & 3.5 & 11.6 & 43.2 \\
\hline $\begin{array}{l}+ \text { alachlor } 0.5 \\
\text { terbuthylazine } 0.9\end{array}$ & 3 & 0 & 2.9 & 4.0 & 10.0 & 41.1 \\
\hline+ alachlor 1.0 & 7 & 0 & 2.6 & 3.7 & 11.8 & 42.2 \\
\hline $\begin{array}{l}\text { terbuthylazine } 1.5 \\
\text { simazine } 1.5\end{array}$ & 5 & 0 & 3.1 & 3.8 & 11.0 & 37.7 \\
\hline $\begin{array}{l}\quad+\text { diuron } 0.5 \\
\text { terbuthylazine } 0.9\end{array}$ & 6 & 0 & 2.6 & 3.9 & 10.0 & 42.9 \\
\hline+ diuron 0.5 & 3 & 0 & 3.2 & 3.7 & 8.9 & 39.2 \\
\hline simazine/haloxyfop 0.25 & 4 & 0 & 2.5 & 3.8 & 10.6 & 38.9 \\
\hline LSD $5 \%$ & 3 & - & $\mathrm{NS}^{7}$ & NS & NS & NS \\
\hline
\end{tabular}

${ }^{1}$ haloxyfop (Gallant). ${ }^{2}$ Plots were scored on a $0-10$ scale, $0=$ no weeds, $1=$ several weeds per plot, $5=10-15 \%$ ground cover, $7=25-30 \%$ weed cover, $10=50 \%$ ground cover (weediest plot). ${ }^{3}$ Clover and nightshade present. ${ }^{4}$ Nightshade present. ${ }^{5}$ Plants were scored on a $0-10$ scale: $0=$ no symptoms, $10=$ all leaves showing severe symptoms. ${ }^{6}$ Plants were scored on a $0-10$ scale: $0=$ no purpling, $10=50 \%$ leaf area purple. ${ }^{7}$ NS Non significant at the $5 \%$ level. 


\section{Trial 3}

All herbicide treatments significantly reduced weed numbers (Table 3). Later in the season, weed growth was greater in this trial than in Trial 2. The herbicide treatments did not significantly affect leaf symptoms, leaf purpling, stem numbers or stem length.

TABLE 3: Trial 3. Weed count on 20 September, weed score on 17 October, leaf symptom score on 21 October, leaf purpling score on 31 January and mean stem number and length in early February.

\begin{tabular}{lrccccc}
\hline $\begin{array}{l}\text { Herbicide treatment } \\
\text { and rate }(\mathrm{kg} \mathrm{ai} / \mathrm{ha})\end{array}$ & $\begin{array}{c}\text { Weed } \\
\text { count } \\
\left(\mathrm{No.} / \mathrm{m}^{2}\right)\end{array}$ & $\begin{array}{c}\text { Weed } \\
\text { score }^{2}\end{array}$ & $\begin{array}{c}\text { Score } \\
\text { for } \\
\text { leaf } \\
\text { symptoms }\end{array}$ & $\begin{array}{c}\text { Leaf } \\
\text { purpling } \\
\text { score }\end{array}$ & $\begin{array}{c}\text { Mean } \\
\text { stem } \\
\text { number } \\
\text { per plant }\end{array}$ & $\begin{array}{c}\text { Mean } \\
\text { stem } \\
\text { length } \\
(\mathrm{cm})\end{array}$ \\
\hline control & 286 & 9.0 & 3.0 & 4.2 & 8.8 & 49.5 \\
propazine 0.9 & 30 & 2.5 & 3.2 & 4.3 & 8.5 & 38.1 \\
propazine 1.5 & 14 & 1.5 & 2.7 & 4.5 & 10.3 & 48.8 \\
bromacil 0.5 + diuron 0.5 & 50 & 3.0 & 3.3 & 4.4 & 7.9 & 42.7 \\
bromacil 0.8 + diuron 0.8 & 6 & 0.5 & 3.5 & 4.2 & 8.2 & 43.1 \\
terbacil 1.0 & 10 & 1.5 & 3.5 & 4.1 & 7.7 & 43.9 \\
oxadiazon 0.9 & 40 & 3.0 & 2.8 & 4.5 & 10.4 & 49.1 \\
oxadiazon 1.5 & 32 & 2.5 & 2.5 & 4.4 & 7.7 & 47.1 \\
dichlobenil 4.0 & 0 & 0.5 & 2.9 & 5.0 & 8.5 & 44.4 \\
LSD 5\% & 159 & 2.4 & $\mathrm{NS}$ & $\mathrm{NS}$ & $\mathrm{NS}$ & $\mathrm{NS}$ \\
\hline
\end{tabular}

${ }^{1}$ propazine (Gesamil 50WP), bromacil + diuron (Krovar 1), terbacil (Sinbar), oxadiazon (Ronstar granules), dichlobenil (Prefix-D granules). ${ }^{2}$ Plots were scored on a $0-10$ scale: $0=$ no weeds, $2=$ several weeds per plot, $8=10 \%$ weed cover, $10=30 \%$ ground cover with weeds. ${ }^{3}$ Plants were scored on a $0-10$ scale: $0=$ no symptoms, 10 $=$ all leaves showing severe symptoms. ${ }^{4}$ Plants were scored on a $0-10$ scale; $0=$ no purpling, $10=50 \%$ leaf area purple.

\section{DISCUSSION}

All treatments tested on a crop recently planted in the field gave good weed control except for alachlor or metolachlor applied alone. Leaf necrosis was increased slightly by several herbicide treatments. However, on established plants (one year after planting in the field - Trial 2) these treatments did not appear to be damaging. This suggests that established gentian crops tolerate herbicides better than newly planted crops.

Persistent herbicides were compared in Trial 3. Surprisingly, no treatment appeared to damage the crop, but dichlobenil and the higher rate of bromacil + diuron were the only treatments that gave weed control nearly comparable with treatments in Trial 2.

This study has identified herbicide mixtures, such as low rates of simazine or terbuthylazine with low rates of alachlor or diuron, that give good weed control and are safe on gentian crops.

\section{REFERENCES}

Barralis, G., Chadoeuf, R. and Desmarest, P., 1978. New trends of Gentiana lutea cultivation. Acta Hort. 73: 303-306.

Batt, P., 1982. Observations of the ornamental industry in northern Japan. Ministry of Agriculture \& Fisheries, Christchurch, New Zealand. 